\title{
COMMENTS ON THE COMPUTATION OF QUANTUM FLUCTUATIONS OF GLUONS IN A MULTI-INSTANTON BACKGROUND
}

\author{
B. BERG and J. STEHR \\ II. Institut für Theoretische Physik, Universität Hamburg, Germany \\ Received 20 March 1980 \\ (Revised 9 June 1980)
}

\begin{abstract}
Relying on previous results, the computation of quantum fluctuations of gluons in a Yang-Mills multi-instanton background field is explained. For the present most comprehensive formula due to Jack the integration constant is determined. The formulae are evaluated in more detail for the special case of the 't Hooft solutions.
\end{abstract}

\section{Introduction}

Let the Dirac operator in an external Yang-Mills gauge field be

$$
\not D=\left(\begin{array}{cc}
0 & D^{+} \\
D & 0
\end{array}\right)
$$

where

$$
D=i e_{\mu}^{+} D_{\mu}, \quad D^{+}=i e_{\mu} D_{\mu}, \quad\left(e_{\mu}\right)=\left(1,-i \sigma_{j}\right)
$$

( $\sigma_{j}$ are the Pauli matrices) and $D_{\mu}=1_{n} \partial_{\mu}+A_{\mu}$ is the covariant derivative. For definiteness we use an $\mathrm{SU}(n)$ gauge group (the extension to other gauge groups is straightforward). By $\varnothing_{0}$ we denote the same operator without gauge field.

Recently, formulae for the determinant of the Dirac operator in a multi-instanton background field $\boldsymbol{A}_{\mu}$ of topological charge $k$ were derived [1] ${ }^{\star}$. In flat space the result for the regularized determinant with zero modes omitted reads ${ }^{\star \star}$

$$
\operatorname{det}^{\prime} \frac{D}{D_{0}}=\sqrt{\operatorname{det}^{\prime} \frac{D^{2}}{D_{0}^{2}}}=\mathrm{e}^{r},
$$

where

$$
\begin{aligned}
\Gamma=\Gamma_{\mathrm{reg}}-\Gamma_{\mathrm{reg}}^{0}= & k\left\{-\frac{2}{3} \ln \mu-4 \zeta^{\prime}(-1)-\frac{2}{3} \ln 2+\frac{5}{12}\right\} \\
& +\frac{1}{24 \pi^{2}} \int \mathrm{d}^{4} x I_{1}(x)+\frac{1}{24 \pi^{2}} \int \mathrm{d}^{4} x \int_{0}^{1} \mathrm{~d} t I_{2}(t, x),
\end{aligned}
$$

* The first paper of [1] is hereafter referred to as I.

* The results of $\mathrm{I}$ were derived for a $\mathrm{Sp}(r)$ gauge group. By imbedding $\mathrm{SU}(n)$ into $\mathrm{Sp}(n)$ it is quite straightforward to show that (2) holds. 
and

$$
\begin{gathered}
I_{1}=\operatorname{Tr}\left\{f \partial_{\mu} f^{-1} f \partial_{\mu} f^{-1} f \partial_{\nu} f^{-1} f \partial_{\nu} f^{-1}\right\} \\
-5 \operatorname{Tr}\{f \underbrace{+} b f \underbrace{+} b\}+4 k\left(1+x^{2}\right)^{-2}, \\
I_{2}=\varepsilon_{\mu \nu \rho \sigma} \operatorname{Tr}\left\{K^{-1} \partial_{t} K K^{-1} \partial_{\mu} K K^{-1} \partial_{\nu} K K^{-1} \partial_{\rho} K K^{-1} \partial_{\sigma} K\right\},
\end{gathered}
$$

with

$$
K(t, x)=\frac{1}{2}(t-1)\left(\lambda^{2}+x^{2}\right) b^{+} b+t f^{-1} .
$$

The $k \times k$ matrix $f$ is related to the instanton parameter matrices of the AtiyahDrinfeld-Hitchin-Manin (ADHM) construction by means of

$$
f^{-1}=-\frac{1}{2} \Delta^{+} \Delta, \quad \Delta_{A^{\prime}}=a_{A^{\prime}}+b^{A} x_{A^{\prime}} .
$$

Here $x=\left(x_{A A^{\prime}}\right)$ is the quaternionic representation of the position vector. The $(n+2 k) \times k$ matrices $a_{A^{\prime}}, b^{A}\left(A^{\prime}=1,2 ; A=1,2\right)$ are the instanton parameter matrices and $\downarrow$ stands for contraction of spinorial indices, e.g., $\Delta^{+} \Delta=\Delta^{+A^{\prime}} \Delta_{A^{\prime}}$. Indices are raised and lowered by $\xi_{A}=\varepsilon_{A B} \xi^{B}$ and the adjoint is in the sense of spinors, i.e., $\Delta_{1}^{+}=-\bar{\Delta}_{2}^{\mathrm{T}}$ and $\Delta_{2}^{+}=\bar{\Delta}_{1}^{\mathrm{T}}$. $\Gamma_{\mathrm{reg}}$ is defined by

$$
\Gamma_{\mathrm{reg}}=\frac{1}{2} \operatorname{Tr}\left\{\ln \left(\not D^{2}+P_{0}\right)+\sum_{i=1}^{\nu} e_{i} \ln \left(D^{2}+M_{i}^{2}\right)\right\},
$$

where $P_{0}$ is the projector onto the zero modes of $D$ and $\ln \mu$ is related to the Pauli-Villars regulators by $\ln \mu=-\sum_{i=1}^{\nu} e_{i} \ln M_{i}$. For more details of the notation cf. I.

In flat space the fluctuation operator for gluons (i.e., of the vector fields in a pure $\mathrm{SU}(n)$ Yang-Mills theory) is trivially related to the Dirac operator in the adjoint $\mathrm{SU}(n)$ representation and a corresponding relation between the determinants of these operators has been assumed in the literature [2]. This conjecture may be supported by an investigation of the gluon determinant on the sphere [3]. We therefore study in the present paper the quantum fluctuation of the Dirac operator in the adjoint $S U(n)$ representation.

The adjoint $S U(n)$ representation can be trivially imbedded into the fundamental $\mathrm{SU}\left(n^{2}\right)$ representation. It follows that special instanton parameter matrices $\tilde{a}_{A^{\prime}}, \tilde{b}^{A}$ of the fundamental $\mathrm{SU}\left(n^{2}\right)$ representation, from which a corresponding $\tilde{f}$ can be constructed by ( $3 \mathrm{~d})$, describe the adjoint $S U(n)$ representation. For the determinant of the Dirac operator formulae (2) and (3) remain true with $f$ replaced by $\tilde{f}$ and $K$ replaced by a corresponding $\tilde{K}$. The matrix $\tilde{f}$ has already been constructed by Corrigan, Goddard and Templeton [4] in their investigation of Green functions in a multi-instanton field of a tensor product representation. In sect. 2 we give the explicit formulae for $\tilde{f}$ in the adjoint $S U(n)$ representation. The thus obtained formulae for the determinant of the Dirac operator in the adjoint $\operatorname{SU}(n)$ representation are 
illustrated in sect. 3 by an explicit evaluation for the one-instanton case and a discussion of the 't Hooft solutions in sect. 4.

Equivalent formulae can be obtained along the lines of [1] by using the Green function [4] of the adjoint representation. This has been done by Jack [5] for tensor product representations. Jack's formula is written down for the adjoint $\mathrm{SU}(n)$ representation at the end of sect. 2 . Compared with the results in terms of $\tilde{f}$, Jack's formula has the great advantage, that the size of the involved matrices is considerably reduced for relevant cases and that one has not to take traces of lengthy matrix expressions. They are therefore more promising than the first formulae for trying a further evaluation. This is illustrated for the ' $t$ Hooft solutions in sect. 4 .

A new integration constant is introduced in the formula of Jack by the method of their computation, whereas no new integration constant is introduced by using the imbedding procedure and $\tilde{f}$. This is employed in sect. 3 , to determine the integration constant (which turns out to be zero). Both formulae suffer at the time from the lack of an unconstrained parametrization of the $\operatorname{SU}(n)$ instantons. This is the reason, why the instanton gas of the non-linear $\sigma$ and $\mathrm{CP}^{N}$ models [6] is still much more explicit than that of the Yang-Mills theory.

\section{Determinant of the Dirac operator in the adjoint $S U(n)$ representation}

Let us consider the Hilbert spaces $\mathrm{H}_{-}\left(\mathrm{H}_{+}\right)$of spinors $\psi_{A}$ of negative $\left(\psi_{A^{\prime}}\right.$ of positive) chirality which take values in the $S U(n)$ Lie algebra. These two spaces are equipped with the scalar product

$$
\begin{aligned}
& (\psi, \phi)=\int \mathrm{d}^{4} x\left\langle\psi_{A}^{+}, \phi^{A}\right\rangle, \quad \psi, \phi \in \mathrm{H}_{-}, \\
& (\psi, \phi)=\int \mathrm{d}^{4} x\left\langle\psi_{A^{\prime}}^{+}, \phi^{A^{\prime}}\right\rangle, \quad \psi, \phi \in \mathrm{H}_{+},
\end{aligned}
$$

where

$$
\langle A, B\rangle=-\frac{1}{2} \operatorname{Tr}(A \cdot B)
$$

for matrices of the $\mathrm{SU}(n)$ Lie algebra.

The Dirac operator in the adjoint $\mathrm{SU}(n)$ representation is given by

$$
\not \nabla=\left(\begin{array}{cc}
0 & \nabla^{+} \\
\nabla & 0
\end{array}\right)
$$

where

$$
\begin{gathered}
\nabla=i e_{\mu}^{+} \nabla_{\mu}, \quad \nabla^{+}=i e_{\mu} \nabla_{\mu}, \\
\nabla_{\mu}=\partial_{\mu}+\left[A_{\mu}, \cdot\right] .
\end{gathered}
$$

$\nabla$ maps negative onto positive chirality spinors and $\nabla^{+}$vice versa. With respect to the scalar product $(4), \nabla^{+}$is the adjoint of $\nabla$. 
We may trivially imbed the adjoint $\mathrm{SU}(n)$ representation into the fundamental $\mathrm{SU}\left(n^{2}\right)$ representation by defining the new gauge field

$$
\tilde{A}_{\mu}=A_{\mu} \otimes 1_{n}+1_{n} \otimes A_{\mu} .
$$

The gauge field $\tilde{A_{\mu}}$ is a multi-instanton solution of topological charge $\tilde{k}=2 n k$.

Let $a(1)_{A^{\prime}}=a_{A^{\prime}}$ and $b(1)^{A}=b^{A}$ be the instanton parameter matrices of the fundamental $\mathrm{SU}(n)$ representation; then the instanton parameter matrices of the conjugate $\mathrm{SU}(n)$ representation are given by

$$
a(2)_{A^{\prime}}=\varepsilon_{A^{\prime} B^{\prime}} \overline{a(1)_{B^{\prime}}}, \quad b(2)^{A}=\varepsilon^{A B} \overline{b(1)^{B}},
$$

where $\varepsilon_{12}=\varepsilon^{12}=1$. Because all instanton fields are obtained by the ADHM construction, there have to be instanton parameter matrices $\tilde{a}_{A^{\prime}}, \tilde{b}^{A}$ which describe $\tilde{A_{\mu}}$. Therefore, the determinant of the Dirac operator in the adjoint $\mathrm{SU}(n)$ representation ${ }^{\star}$ is obtained by replacing in (3) $b$ by $\tilde{b}, f$ by $\tilde{f}$ and correspondingly $K$ by

$$
\tilde{K}=\frac{1}{2}(t-1)\left(\lambda^{2}+x^{2}\right) \tilde{b}^{+} \tilde{b}+t \tilde{f}^{-1} .
$$

In the present notation the recipe [4] for constructing $\tilde{f}$ is given in the following. Up to a transformation $\tilde{f} \rightarrow K^{+} \tilde{f} K$ with an element $K \in \mathrm{Gl}(\tilde{k}, \mathbb{C}) \tilde{f}$ is given by

$$
\tilde{f}^{-1}=\frac{1}{2} Z^{+} \Omega^{-1} Z \text {, }
$$

where

$$
\begin{gathered}
Z_{r s}=\operatorname{Tr}\left(c_{r}^{+} c_{s}\right)+\operatorname{Tr}\left(d_{r}^{+} d_{s}\right), \\
\Omega_{r s}=4\left\{\operatorname{Tr}\left(c_{r}^{+} c_{s} \bar{f}\right)+\operatorname{Tr}\left(d_{r}^{+} d_{s} f\right)-\operatorname{Tr}\left(\tilde{f} c_{r}^{+} \Delta f \Delta^{+} c_{s}\right)\right\} .
\end{gathered}
$$

The matrices $c_{s}, d_{s}(s=1, \ldots, \tilde{k})$ are a basis of $(n+2 k) \times k$ matrices, which are defined by the relation

$$
\left(\Delta_{A^{\prime}}^{+}(1) c\right)_{i j}=\left(\Delta_{A^{\prime}}^{+}(2) d\right)_{j i}, \quad A^{\prime}=1,2,
$$

where

$$
\Delta_{A^{\prime}}^{+}(i)=a^{+}(i)_{A^{\prime}}+b^{+}(i)^{A} x_{A^{\prime}}, \quad i=1,2 .
$$

$\tilde{b}^{+} \tilde{b}$ is obtained by means of

$$
\tilde{b}^{+} \tilde{b}=-Z^{+} \Omega_{b}^{-1} Z
$$

with

$\left(\Omega_{b}\right)_{r s}=-8\left\{\operatorname{Tr}\left(c_{r}^{+} c_{s}\left(\underline{b}^{+} b\right)^{-1}\right)+\operatorname{Tr}\left(d_{r}^{+} d_{s}\left(\underline{b}^{+} b\right)^{-1}\right)+2 \operatorname{Tr}\left(\left(\bar{b}^{+} b\right)^{-1} c_{r}^{+} b\left(\underline{b}^{+} b\right)^{-1} b^{+} c_{s}\right)\right\}$.

In sect. 3 we will illustrate the obtained formulae for special examples.

* It is easily seen that the singlet part does not contribute. 
Equivalent formulae can be derived along the lines of [1], by using the Green function [4] for tensor product representations. This has been done by Jack [5]. For the Dirac operator in the adjoint $S U(n)$ representation his result would become

$$
\operatorname{det}^{\prime} \frac{\not \nabla}{\not_{0}} \stackrel{\text { def }}{=} \sqrt{\operatorname{det}^{\prime} \frac{\nabla^{2}}{\not_{0}^{2}}}=\mathrm{e}^{\tilde{\Gamma}}
$$

with

$$
\begin{aligned}
\tilde{\Gamma}= & 2 n \Gamma-2 \ln \operatorname{det}\left[\frac{1}{4} M\left(b^{+} b \otimes b^{+} b\right)\right] \\
& +\frac{1}{8 \pi^{2}} \int \mathrm{d}^{4} x \ln \operatorname{det}\left[-\frac{1}{2} f b^{+} b\right] \square^{2} \ln \operatorname{det}\left[-\frac{1}{2} f b^{+} b\right]+\tilde{k} \cdot c .
\end{aligned}
$$

Here $c$ is an unknown constant and $M$ is the conformal invariant matrix $[10,4]$ given by

$$
\begin{aligned}
M^{-1}= & \frac{1}{4} a^{+}(1) a(1) \otimes b^{+}(2) b(2)+\frac{1}{4} b^{+}(1) b(1) \otimes a^{+}(2) a(2) \\
& -a^{+}(1) b(1) \otimes a^{+}(2) b(2) .
\end{aligned}
$$

\section{The one-instanton case}

We now evaluate explicitly the one-instanton case, using (2), (3) with $f$ replaced by $\tilde{f}$, and (7), (8). We only consider the $\mathrm{SU}(2)$ one-instanton solution, the $\mathrm{SU}(n)$ one-instanton solution can be obtained by trivial imbedding.

The SU(2) one-instanton solution is given by

$$
A_{\mu}=\frac{1}{2} \bar{\sigma}_{\mu \nu} \partial_{\nu} \ln \rho, \quad \rho=1+\frac{\lambda^{2}}{(x-y)^{2}},
$$

and $\bar{\sigma}_{\mu \nu}=e_{\mu}^{+} e_{\nu}-\delta_{\mu \nu} y$ is the position and $\lambda>0$ the scale size of the instanton. The corresponding instanton parameter matrices of the ADHM construction are

$$
a_{A^{\prime}}=\left(\begin{array}{c}
\lambda \delta_{1 A^{\prime}} \\
\lambda \delta_{2 A^{\prime}} \\
-y_{1 A^{\prime}} \\
-y_{2 A^{\prime}}
\end{array}\right), \quad b^{A}=\left(\begin{array}{c}
0 \\
0 \\
\delta^{1 A} \\
\delta^{2 A}
\end{array}\right) .
$$

The indices of $y$ refer to its quaternionic representation. The matrices $\Delta_{A^{\prime}}=$ $a_{A^{\prime}}+b^{A} x_{A^{\prime}}$ are

$$
\Delta_{A^{\prime}}=\left(\begin{array}{c}
\lambda \delta_{1 A^{\prime}} \\
\lambda \delta_{2 A^{\prime}} \\
(x-y)_{1 A^{\prime}} \\
(x-y)_{2 A^{\prime}}
\end{array}\right)
$$


and it follows $f^{-1}=\lambda^{2}+(x-y)^{2}$. In the following we exploit euclidean covariance and choose $y=0$. The full formulae are obtained by the substitution $x \rightarrow x-y$.

A convenient basis for the matrices $\left(c_{r}, d_{r}\right)$, defined by $(9)$ is

$$
c_{r}=\left(\begin{array}{c}
\delta_{r 1} \\
\delta_{r 2} \\
\delta_{r 3} \\
\delta_{r 4}
\end{array}\right), \quad d_{r}=\left(\begin{array}{c}
-\delta_{r 2} \\
\delta_{r 1} \\
-\delta_{r 4} \\
\delta_{r 3}
\end{array}\right) .
$$

Using formulae (8), (10) it is not difficult to compute $\tilde{f}$ and $\tilde{b}^{+} \tilde{b}$. The result is conveniently expressed in the form of $2 \times 2$ matrices of quaternions:

$$
\begin{gathered}
\tilde{f}=2 f^{2}\left(\begin{array}{cc}
\lambda^{2}+2 x^{2} & -\lambda x^{+} \\
-\lambda x & 2 \lambda^{2}+x^{2}
\end{array}\right), \\
\tilde{f}^{-1}=\frac{1}{4}\left(\begin{array}{cc}
2 \lambda^{2}+x^{2} & \lambda x^{+} \\
\lambda x & \lambda^{2}+2 x^{2}
\end{array}\right), \\
\tilde{b}^{+} b=-\frac{1}{2}\left(\begin{array}{ll}
1 & 0 \\
0 & 2
\end{array}\right) .
\end{gathered}
$$

Remember that the gauge field corresponding to $\tilde{f}$ has topological charge $\tilde{k}=4$.

We use these formulae to calculate the integrals in (2). $\tilde{K}$ is obtained from (3c). After some calculation the traces [cf. (3a), (3b)] become

$$
\begin{aligned}
\tilde{I}_{1}= & \frac{64 x^{12}+352 \lambda^{2} x^{10}+784 \lambda^{4} x^{8}+896 \lambda^{6} x^{6}+544 \lambda^{8} x^{4}+160 \lambda^{10} x^{2}+16 \lambda^{12}}{\left(x^{2}+\lambda^{2}\right)^{8}} \\
& -\frac{80 x^{4}+240 \lambda^{2} x^{2}+170 \lambda^{4}}{\left(x^{2}+\lambda^{2}\right)^{4}}+\frac{16}{\left(1+x^{2}\right)^{2}}, \\
\tilde{I}_{2}= & \left(c_{5} x^{10}+c_{4} x^{8}+c_{3} x^{6}+c_{2} x^{4}+c_{1} x^{2}+c_{0}\right) \cdot \tilde{N}^{-1},
\end{aligned}
$$

where

$$
\begin{aligned}
& c_{5}=4 \lambda^{2} t^{4}, \\
& c_{4}=4 \lambda^{8} t^{4}\left(-t^{2}+t+5\right), \\
& c_{3}=\lambda^{10} t^{4}\left(t^{4}-2 t^{3}-15 t^{2}+16 t+40\right), \\
& c_{2}=\lambda^{12} t^{4}\left(3 t^{4}-6 t^{3}-21 t^{2}+24 t+40\right), \\
& c_{1}=\lambda^{14} t^{4}\left(3 t^{4}-6 t^{3}-13 t^{2}+16 t+20\right), \\
& c_{0}=\lambda^{16} t^{4}\left(t^{4}-2 t^{3}-3 t^{2}+4 t+4\right), \\
& \tilde{N}=\frac{2}{9}\left(x^{2}+\lambda^{2}\right)^{5}\left(x^{2}+z^{2}\right)^{5} \\
& z^{2}=\frac{1}{2} \lambda^{2}(1+t)(2-t)
\end{aligned}
$$


Doing even more calculations, the integrals are found to be

$$
\begin{array}{r}
\frac{1}{24 \pi^{2}} \int \mathrm{d}^{4} x \tilde{I}_{1}=-\frac{181}{72}+\frac{4}{3} \ln \lambda, \\
\frac{1}{24 \pi^{2}} \int \mathrm{d}^{4} x \int_{0}^{1} \mathrm{~d} t \tilde{I}_{2}=2 \ln 2-\frac{11}{8} .
\end{array}
$$

It is amazing to note that in this formulation the 5-dimensional integral already contributes to the one-instanton fluctuations. This should be compared with the fact that for SU(2) a consequence of Jack's result is a formula which does not involve a five-dimensional integral at all. Collecting all contributions we arrive at

$$
\tilde{\Gamma}=-\frac{8}{3} \ln \mu-16 \zeta^{\prime}(-1)-\frac{2}{3} \ln 2+\frac{4}{3} \ln \lambda-\frac{20}{9} .
$$

This result is consistent with previous literature [7]. It is an easy matter to evaluate (11) for the one-instanton case. Comparison with (16) determines the constant $c$ to be

$$
c=0 \text {. }
$$

\section{The 't Hooft solution}

The evaluation of $\tilde{\Gamma}$ using (7)-(10) is straightforward but very cumbersome, as is already seen for the 't Hooft solution

$$
A_{\mu}=\frac{1}{2} \bar{\sigma}_{\mu \nu} \partial_{\nu} \ln \rho, \quad \rho=1+\sum_{i=1}^{k} \frac{\lambda_{i}^{2}}{\left(x-y^{i}\right)^{2}}
$$

in the two-instanton case $(k=2)$.

Then

$$
a_{A^{\prime}}=\left(\begin{array}{cc}
\lambda_{1} \delta_{1 A^{\prime}} & \lambda_{2} \delta_{1 A^{\prime}} \\
\lambda_{1} \delta_{2 A^{\prime}} & \lambda_{2} \delta_{2 A^{\prime}} \\
-y_{1 A^{\prime}}^{1} & 0 \\
-y_{2 A^{\prime}}^{1} & 0 \\
0 & -y_{1 A^{\prime}}^{2} \\
0 & -y_{2 A^{\prime}}^{2}
\end{array}\right), \quad b^{A}=\left(\begin{array}{cc}
0 & 0 \\
0 & 0 \\
\delta^{1 A} & 0 \\
\delta^{2 A} & 0 \\
0 & \delta^{1 A} \\
0 & \delta^{2 A}
\end{array}\right) \text {. }
$$

From (9) follows

$$
c=\left(\begin{array}{ll}
\gamma_{1} & \gamma_{3} \\
\gamma_{2} & \gamma_{4} \\
\gamma_{5} & u_{1} \\
\gamma_{6} & u_{2} \\
u_{1} & \gamma_{7} \\
u_{2} & \gamma_{8}
\end{array}\right), \quad d=\left(\begin{array}{cc}
-\gamma_{2} & -\gamma_{4} \\
\gamma_{1} & \gamma_{3} \\
-\gamma_{6} & -u_{2} \\
\gamma_{5} & u_{1} \\
-u_{2} & -\gamma_{8} \\
u_{1} & \gamma_{7}
\end{array}\right)
$$


with

$$
u_{A}=\frac{1}{\left(y^{1}-y^{2}\right)^{2}}\left\{\left(y^{1}-y^{2}\right)_{A 1^{\prime}}\left(\lambda_{1} \gamma_{3}-\lambda_{2} \gamma_{1}\right)+\left(y^{1}-y^{2}\right)_{A 2^{\prime}}\left(\lambda_{1} \gamma_{4}-\lambda_{2} \gamma_{2}\right)\right\}
$$

and arbitrary $\gamma_{i}$. Setting $\gamma_{i}=\delta_{i s}$ we get a basis of matrices $c_{s}$ and $d_{s}$ in which the $8 \times 8$ matrices $\tilde{f}, \tilde{f}^{-1}$ and $\tilde{b}^{+} \tilde{b}$ can be expressed as $4 \times 4$ matrices of quaternions. The results are stated in appendix B. They are much more complicated than the corresponding $f$, $f^{-1}$ in the fundamental representation.

For the evaluation of $\tilde{\Gamma}$ in the multi-instanton case formula (11) is more convenient. Casting $b^{A}$ into normal form, i.e., $b^{+} b=-2$, we have (cf. formula (56.16) of [5] or (73) of I)

$$
\Gamma=-k\left\{\frac{2}{3} \ln \mu+4 \zeta^{\prime}(-1)+\ln 2\right\}+\frac{1}{3} \ln \operatorname{det} M_{\mathrm{S}}^{-1}+\frac{1}{96 \pi^{2}} \int \mathrm{d}^{4} x \ln \operatorname{det} f^{-1} \square^{2} \ln \operatorname{det} f^{-1}
$$

[where $\operatorname{det} M_{\mathrm{S}}^{-1}$ is given by (26)] and

$$
\tilde{\Gamma}=4 \Gamma+2 \ln \operatorname{det} M^{-1}+\frac{1}{8 \pi^{2}} \int \mathrm{d}^{4} x \ln \operatorname{det} f^{-1} \square^{2} \ln \operatorname{det} f^{-1} .
$$

For the $k$-instanton ' $t$ Hooft solution we generalize (18) and find

$$
\begin{gathered}
f_{i j}^{-1}=\lambda_{i} \lambda_{i}+\left(x-y^{i}\right)^{2} \delta_{i j}, \quad i, j=1, \ldots, k, \\
M_{i l, j m}^{-1}=\lambda_{i} \lambda_{j} \delta_{l m}+\lambda_{1} \lambda_{m} \delta_{i j}+\left(y^{i}-y^{l}\right)^{2} \delta_{i j} \delta_{l m}, \quad i, j, m=1, \ldots, k
\end{gathered}
$$

Noting that

$$
\hat{f}_{i j}^{-1} \stackrel{\text { def }}{=} \frac{\lambda_{i}}{\sqrt{\left(x-y^{i}\right)^{2}}} \cdot \frac{\lambda_{i}}{\sqrt{\left(x-y^{j}\right)^{2}}}+\delta_{i j}
$$

is a unit matrix plus a matrix of rank 1 with

$$
\operatorname{det} \hat{f}^{-1}=\rho,
$$

we have

$$
\operatorname{det} f^{-1}=\rho \prod_{i=1}^{k}\left(x-y^{i}\right)^{2}=\tilde{\rho} .
$$

Corrigan et al. [1] give an expression for $\operatorname{det}\left(M^{-1}\right)$ in a different parametrization [11] for the $k$-instanton 't Hooft solution. Since we need $\operatorname{det}\left(\boldsymbol{M}_{\mathrm{S}}^{-1}\right)$ and $\operatorname{det}\left(\boldsymbol{M}_{\mathrm{A}}^{-1}\right)$ separately, we give a derivation which is quite different. The result consists of graphical rules to write $\operatorname{down} \operatorname{det}\left(\boldsymbol{M}_{\mathrm{A}}^{-1}\right)$ as a function of the instanton parameters. We see no way to derive these graphical rules directly from the rules for the evaluation of det $\left(M^{-1}\right)$ given by Corrigan et al. (adjusted to our parametrization). $M^{-1}$ can be factorised into one part $M_{\mathrm{S}}^{-1}$ which acts only on the symmetric part of the 
tensor product space and another part $M_{\mathrm{A}}^{-1}$ which acts on the antisymmetric part of the tensor product space:

$$
\begin{aligned}
& M_{\mathrm{S} i l, j m}^{-1}=M_{i l, j m}^{-1}+M_{i l, m j}^{-1}-M_{i l, j i}^{-1} \delta_{j m}, \quad i \geqslant l, j \geqslant m, \\
& M_{\mathrm{A} i l, j m}^{-1}=M_{i l, j m}^{-1}-M_{i l, m j}^{-1}, \quad i>l, j>m,
\end{aligned}
$$

and

$$
\operatorname{det} M^{-1}=\operatorname{det} M_{\mathrm{S}}^{-1} \cdot \operatorname{det} M_{\mathrm{A}}^{-1} \text {. }
$$

Inserting (22) yields

$$
\begin{aligned}
& M_{\mathrm{S} i l, j m}^{-1}=\left(1-\delta_{i l}\right)\left(1-\delta_{j m}\right)\left[\lambda_{i} \lambda_{j} \delta_{l m}+\lambda_{l} \lambda_{m} \delta_{i j}+\lambda_{i} \lambda_{m} \delta_{l j}+\lambda_{l} \lambda_{j} \delta_{i m}+\left(y^{i}-y^{l}\right)^{2} \delta_{i j} \delta_{l m}\right] \\
&+\left(1-\delta_{i l}\right) \delta_{j m}\left[\lambda_{i} \lambda_{j} \delta_{l i}+\lambda_{l} \lambda_{j} \delta_{i j}\right]+\delta_{i l} \delta_{i m}\left[2 \lambda_{i}^{2} \delta_{i j}\right] \\
&+\delta_{i l}\left(1-\delta_{j m}\right)\left[2 \lambda_{i} \lambda_{j} \delta_{i m}+2 \lambda_{i} \lambda_{m} \delta_{i j}\right], \\
& k \geqslant i \geqslant l \geqslant 1, \quad k \geqslant j \geqslant m \geqslant 1 \\
& M_{\mathrm{A} i l, j m}^{-1}=\lambda_{i} \lambda_{j} \delta_{l m}+\lambda_{l} \lambda_{m} \delta_{i j}-\lambda_{i} \lambda_{m} \delta_{l j}-\lambda_{l} \lambda_{j} \delta_{i m}+\left(y^{i}-y^{l}\right)^{2} \delta_{i j} \delta_{l m} \\
& k \geqslant i>l \geqslant 1, \quad k \geqslant j>m \geqslant 1 .
\end{aligned}
$$

By the elementary row transformation for $i>l$,

$$
\text { row } i l \rightarrow \text { row } i l-\frac{\lambda_{l}}{2 \lambda_{i}} \text { row } i i-\frac{\lambda_{i}}{2 \lambda_{l}} \text { row } l l \text {, }
$$

$M_{\mathrm{S}}^{-1}$ is brought to triangular form and we read off

$$
\operatorname{det} M_{\mathrm{S}}^{-1}=2^{k} \prod_{i=1}^{k} \lambda_{i}^{2} \prod_{r>s}\left(y^{r}-y^{s}\right)^{2}
$$

The computation of $\operatorname{det} M_{\mathrm{A}}^{-1}$ is not so easy. For a complete result see appendix $\mathrm{A}$. Here we only give a formula, which is exact for $k \leqslant 3$. For $k>3$ it describes the leading behaviour when the instantons are far apart from each other compared to their scale size $\lambda_{i}$ :

$$
\operatorname{det} M_{\mathrm{A}}^{-1}=R(k) \prod_{r>s}\left(y^{r}-y^{s}\right)^{2}
$$

with

$$
\begin{aligned}
R(k)= & 1+\frac{1}{2} \sum_{i, j=1}^{k} \frac{\lambda_{i}^{2}+\lambda_{j}^{2}}{\left(y^{i}-y^{j}\right)^{2}}+\frac{1}{2} \sum_{i, j, l=1}^{k} \frac{\left(\lambda_{i}^{2}+\lambda_{j}^{2}+\lambda_{l}^{2}\right) \lambda_{j}^{2}}{\left(y^{i}-y^{j}\right)^{2}\left(y^{j}-y^{l}\right)^{2}} \\
& +\frac{1}{8} \sum_{i, j, l, m=1}^{k} \frac{\left(\lambda_{i}^{2}+\lambda_{j}^{2}\right)\left(\lambda_{l}^{2}+\lambda_{m}^{2}\right)}{\left(y^{i}-y^{j}\right)^{2}\left(y^{l}-y^{m}\right)^{2}}+\cdots
\end{aligned}
$$

The prime on the multiple summations means that terms where two indices are equal should be omitted. Note that $n$-fold sums only contribute for $k \geqslant n$. 
Collecting our results we arrive at

$$
\begin{aligned}
\Gamma= & -k\left[\frac{2}{3} \ln \mu+4 \zeta^{\prime}(-1)+\frac{2}{3} \ln 2\right]+\frac{2}{3} \sum_{i=1}^{k} \ln \lambda_{i}+\frac{1}{3} \sum_{r>s} \ln \left(y^{r}-y^{s}\right)^{2} \\
& +\frac{1}{96 \pi^{2}} \int \mathrm{d}^{4} x \ln \tilde{\rho} \square^{2} \ln \tilde{\rho} \\
\tilde{\Gamma}=4 \Gamma+ & \frac{1}{8 \pi^{2}} \int \mathrm{d}^{4} x \ln \tilde{\rho} \square^{2} \ln \tilde{\rho}+2 k \ln 2 \\
& +4 \sum_{i=1}^{k} \ln \lambda_{i}+4 \sum_{r>s} \ln \left(y^{r}-y^{s}\right)^{2}+2 \ln R(k) \\
= & -4 k\left[\frac{2}{3} \ln \mu+4 \zeta^{\prime}(-1)+\frac{1}{6} \ln 2\right]+\frac{20}{3} \sum_{i=1}^{k} \ln \lambda_{i}+\frac{16}{3} \sum_{r>s} \ln \left(y^{r}-y^{s}\right)^{2} \\
& +\frac{1}{6 \pi^{2}} \int \mathrm{d}^{4} x \ln \tilde{\rho} \square^{2} \ln \tilde{\rho}+2 \ln R(k)
\end{aligned}
$$

These formulae complete the early work of Brown and Creamer [8].

We thank M. Lüscher for useful discussions, $P$. Weisz for reading the manuscript and the referee for several useful remarks.

\section{Appendix A}

EVALUATION OF $\operatorname{det} M_{\mathrm{A}}^{-1}$

From

$$
\begin{gathered}
M_{\mathrm{A}}^{-1}(k)_{i l, j m}=\lambda_{i} \lambda_{j} \delta_{l m}+\lambda_{l} \lambda_{m} \delta_{i j}-\lambda_{i} \lambda_{m} \delta_{l j}-\lambda_{l} \lambda_{j} \delta_{i m}+\left(y^{i}-y^{l}\right) \delta_{i j} \delta_{l m}, \\
k \geqslant i>1, \quad k \geqslant j>m \geqslant 1,
\end{gathered}
$$

we see:

(i) Det $M_{\mathrm{A}}^{-1}(k)$ is a polynomial in $\left(y^{r}-y^{s}\right)^{2}$ and $\lambda_{i}^{2}$ which is symmetric under the interchange of any two indices. Each term of the polynomial contains $n$ factors $\left(y^{r}-y^{s}\right)^{2}$ and $\hat{k}-n$ factors $\lambda_{i}^{2}$ with $\hat{k}=\frac{1}{2} k(k-1)$ the dimension of the tensor product space on which $M_{\mathrm{A}}^{-1}(k)$ acts.

(ii) If all $\left(y^{r}-y^{s}\right)^{2}$ vanish, the rank of $M_{\mathrm{A}}^{-1}$ is $k-1$, as one sees by choosing as an orthogonal basis $\omega^{(r, s)}$ for the tensor product space on which $M_{\mathrm{A}}^{-1}$ acts:

$$
\omega_{j m}^{(r, s)}=\lambda_{j}^{(r)} \lambda_{m}^{(s)}-\lambda_{m}^{(r)} \lambda_{j}^{(s)}, \quad r>s,
$$

with

$$
\sum_{j=1}^{k} \lambda_{j}^{(r)} \lambda_{j}^{(s)}=\delta^{r s} \sum_{j=1}^{k}\left(\lambda_{j}^{(r)}\right)^{2}, \quad \lambda_{j}^{(1)}=\lambda_{j}
$$


Thus for $\operatorname{det} M_{\mathrm{A}}^{-1}(k)$ to be non-vanishing, it is necessary that at least $\hat{k}-(k-1)$ different $\left(y^{r}-y^{s}\right)^{2}$ are not equal to zero. It follows that each term in the polynomial must contain at least $\hat{k}-(k-1)$ factors $\left(y^{r}-y^{s}\right)^{2}$, i.e., at most $k-1$ factors $\lambda_{i}^{2}$.

Now for $\lambda_{k}=0, \operatorname{det} M_{\mathrm{A}}^{-1}(k)$ factorizes according to

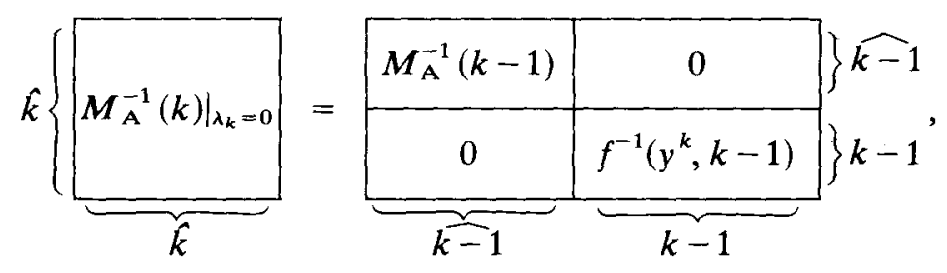

which means, see (23),

$$
\operatorname{det} M_{\mathrm{A}}^{-1}(k)=\operatorname{det} M_{\mathrm{A}}^{-1}(k-1) \cdot \tilde{\rho}\left(y^{k}, k-1\right)+\lambda_{k}^{2} \cdot g,
$$

with unknown $g$. Because of the symmetry property (i) we can repeat this procedure for any $\lambda_{i}, i=1, \ldots, k-1$. Comparing the different expressions for $\operatorname{det} M_{\mathrm{A}}^{-1}(k)$ found in this way, we see that $\operatorname{det} M_{\mathrm{A}}^{-1}(k)$ is determined up to a term of the form $\hat{g} \prod_{i=1}^{k} \lambda_{i}^{2}$. But this term must vanish because it contains too many factors of $\lambda_{i}^{2}$ accoring to (ii).

Setting

$$
\operatorname{det} M_{\mathrm{A}}^{-1}(k)=R(k) \prod_{i>j}\left(y^{i}-y^{j}\right)^{2},
$$

we find that $R(k)$ is determined by the following requirements:

(i) $R(k)$ is a dimensionless polynomial in $\lambda_{i}^{2}$ and $\left(y^{r}-y^{s}\right)^{-2}$ which is symmetric under the interchange of any two indices. The highest power of $\lambda_{i}^{2}$ is $k-1$.

(ii) $\left.\quad R(k)\right|_{\lambda_{k}=0}=R(k-1) \cdot \rho\left(y^{k}, k-1\right)=R(k-1)\left\{1+\sum_{i=1}^{k-1} \frac{\lambda_{j}^{2}}{\left(y^{k}-y^{j}\right)^{2}}\right\}$.

(iii) $\quad R(1)=1$.

The solution is conveniently stated in graph theoretical language [9].

$\boldsymbol{R}(k)$ can be expressed as the sum of all possible unoriented graphs with $k$ vertices (labelled $r=1, \ldots, k$ ) and an arbitrary number of links, such that no closed paths are formed. There is no restriction on the number of links attached to one vertex, but the total number of links in a graph is less than $k$ due to the absence of closed loops. The graphs are evaluated according to the following rules:

each vertex $r$ gives a factor $\lambda_{r}^{-2}$;

each link (connecting vertex $r$ with vertex $s$ ) gives a factor $\lambda_{r}^{2} \lambda_{s}^{2}\left(y^{r}-y^{s}\right)^{-2}$;

each set $\mathrm{S}$ of connected vertices gives a factor $\sum_{i \in \mathrm{S}} \lambda_{i}^{2}$.

Immediate consequences of these rules are:

each isolated vertex gives just a factor 1 ;

each vertex $r$ with $n \geqslant 1$ links attached to it gives a factor $\lambda_{r}^{2(n-1)}$;

the contribution of a connected part of a graph is dimensionless. 
One easily sees that requirements (i) and (iii) are met. Realising that for $\lambda_{k}=0$ only those graphs give a non-vanishing contribution, which have not more than one link attached to vertex $k$, one also sees that requirement (ii) is fulfilled.

The formula (27b) given in the text was gained by adding all graphs with not more than two links. The numerical factors in front of the sums compensate double counting.

\section{Appendix B}

THE MATRIX ELEMENTS OF $\tilde{f}, \tilde{f}^{-1}$, AND $\tilde{b}^{+} \dot{b}$ IN THE TWO-INSTANTON CASE

We choose our basis of matrices $c_{r}, d_{r}$ [cf. (19)] in such a way that the matrix elements of $\tilde{f}, \tilde{f}^{-1}$ and $\tilde{b}^{+} \tilde{b}$ have some useful relations among each other:

(i) $\tilde{f}, \tilde{f}^{-1}, \tilde{b}^{+} \tilde{b}$ are self-adjoint (for all choices of $c_{r}, d_{r}$ );

(ii) they can be expressed as $4 \times 4$ matrices of quaternions and we use the convention

$$
x^{+} \cdot x=x \cdot x^{+}=x_{\mu} x_{\mu} 1_{2}=x^{2}
$$

(iii) let $m$ be one of the matrices $\tilde{f}, \tilde{f}^{-1}, \tilde{b}^{+} \tilde{b}$ : then the quaternionic matrix elements

$$
m\left(\lambda_{1}, \lambda_{2}, y^{1}, y^{2}\right)_{i j}, \quad i, j=1, \ldots, 4,
$$

fulfill

$$
\begin{aligned}
m\left(\lambda_{1}, \lambda_{2}, y^{1}, y^{2}\right)_{2 r, 2 s} & =m\left(\lambda_{2}, \lambda_{1}, y^{2}, y^{1}\right)_{2 r-1,2 s-1}, \\
m\left(\lambda_{1}, \lambda_{2}, y^{1}, y^{2}\right)_{2 r, 2 s-1} & =m\left(\lambda_{2}, \lambda_{1}, y^{2}, y^{1}\right)_{2 r-1,2 s},
\end{aligned}
$$

for $r=1,2 ; s=1,2$.

Therefore it is sufficient that here we only list the quaternionic matrix elements $m_{11}, m_{12}, m_{13}, m_{14}, m_{33}, m_{34}$. Using the following abbreviations:

$$
\begin{aligned}
& \tilde{\rho}=\left(x-y^{1}\right)^{2}\left(x-y^{2}\right)^{2}+\lambda_{1}^{2}\left(x-y^{2}\right)^{2}+\lambda_{2}^{2}\left(x-y^{1}\right)^{2}, \\
& \sigma=\left(y^{1}-y^{2}\right)^{2}+2 \lambda_{1}^{2}+2 \lambda_{2}^{2}, \\
& \tau=\left(y^{1}-y^{2}\right)^{2}+\lambda_{1}^{2}+\lambda_{2}^{2},
\end{aligned}
$$

we obtain the following.

Matrix elements of $\tilde{f}$ :

$$
\begin{aligned}
\sigma^{2} \tilde{\rho}^{2} \tilde{f}_{11}= & 2 \lambda_{2}^{2}\left(\tilde{\rho}+\lambda_{1}^{2} \lambda_{2}^{2}\right)\left(y^{1}-y^{2}\right)^{2}\left[\left(y^{1}-y^{2}\right)^{2}-2\left(x-y^{1}\right)^{2}-2\left(x-y^{2}\right)^{2}\right] \\
& +2 \sigma\left(y^{1}-y^{2}\right)^{2}\left(\lambda_{2}^{2}+\left(x-y^{2}\right)^{2}\right)\left[\tilde{\rho}+\left(x-y^{1}\right)^{2}\left(x-y^{2}\right)^{2}\right] \\
& +4\left(\sigma-2 \lambda_{2}^{2}\right) \tilde{\rho}\left[\tilde{\rho}-\left(x-y^{1}\right)^{2}\left(x-y^{2}\right)^{2}\right] \\
& +4 \lambda_{1}^{2} \sigma\left(x-y^{1}\right)^{2}\left(x-y^{2}\right)^{4}
\end{aligned}
$$




$$
\begin{aligned}
\sigma^{2} \tilde{\rho}^{2} \tilde{f}_{12}= & -2 \lambda_{1} \lambda_{2}\left(\tilde{\rho}+\lambda_{1}^{2} \lambda_{2}^{2}\right)\left(y^{1}-y^{2}\right)^{2}\left[\left(y^{1}-y^{2}\right)^{2}-2\left(x-y^{1}\right)^{2}-2\left(x-y^{2}\right)^{2}\right] \\
& -2 \lambda_{1} \lambda_{2} \sigma \tilde{\rho}\left(y^{1}-y^{2}\right)^{2}+8 \lambda_{1} \lambda_{2} \tilde{\rho}\left[\tilde{\rho}-\left(x-y^{1}\right)^{2}\left(x-y^{2}\right)^{2}\right] \\
& -4 \lambda_{1} \lambda_{2} \sigma\left(x-y^{1}\right)^{2}\left(x-y^{2}\right)^{2}\left(x-y^{2}\right)^{+}\left(x-y^{1}\right), \\
\sigma \tilde{\rho}^{2} \tilde{f}_{13}= & -2 \lambda_{1}\left(y^{1}-y^{2}\right)^{2}\left(\lambda_{2}^{2}+\left(x-y^{2}\right)^{2}\right)^{2}\left(x-y^{1}\right)^{+} \\
& -4 \lambda_{1}^{3}\left(x-y^{2}\right)^{4}\left(x-y^{1}\right)^{+}+4 \lambda_{1}^{3} \lambda_{2}^{2}\left(x-y^{2}\right)^{2}\left(y^{1}-y^{2}\right)^{+}, \\
\sigma \tilde{\rho}^{2} \tilde{f}_{14}= & 2 \lambda_{1} \lambda_{2}^{2}\left(y^{1}-y^{2}\right)^{2}\left(\lambda_{1}^{2}+2\left(x-y^{1}\right)^{2}\right)\left(x-y^{2}\right)^{+} \\
& -4 \lambda_{1} \lambda_{2}^{2}\left(x-y^{1}\right)^{4}\left(x-y^{2}\right)^{+}+4 \lambda_{1} \lambda_{2}^{4}\left(x-y^{1}\right)^{2}\left(y^{1}-y^{2}\right)^{+}, \\
\tilde{\rho}^{2} \tilde{f}_{33}= & 2\left(\lambda_{2}^{2}+\left(x-y^{2}\right)^{2}\right)\left(\tilde{\rho}+\lambda_{1}^{2}\left(x-y^{2}\right)^{2}\right), \\
\tilde{\rho}^{2} \tilde{f}_{34}= & -2 \lambda_{1}^{2} \lambda_{2}^{2}\left(x-y^{1}\right)\left(x-y^{2}\right)^{+} .
\end{aligned}
$$

Matrix elements of $\tilde{f}^{-1}$ (note that $\tilde{f}^{-1}$ is indeed a quadratic polynomial in $x$ ):

$$
\begin{aligned}
4 \tau\left(y^{1}-y^{2}\right)^{2} \tilde{f}_{11}^{-1}= & \left(\sigma-\lambda_{1}^{2}\right)\left(\sigma-2 \lambda_{1}^{2}\right)\left(x-y^{1}\right)^{2}+2 \lambda_{1}^{2} \lambda_{2}^{2}\left(x-y^{2}\right)^{2} \\
& +2 \lambda_{1}^{2}\left(\sigma-\lambda_{1}^{2}\right)\left(y^{1}-y^{2}\right)^{2}+4 \lambda_{1}^{2} \lambda_{2}^{4}, \\
4 \tau\left(y^{1}-y^{2}\right)^{2} \tilde{f}_{12}^{-1}= & -\lambda_{1} \lambda_{2}\left(\sigma-2 \lambda_{1}^{2}\right)\left(x-y^{1}\right)^{2}-\lambda_{1} \lambda_{2}\left(\sigma-2 \lambda_{2}^{2}\right)\left(x-y^{2}\right)^{2} \\
& +\lambda_{1} \lambda_{2}\left(y^{1}-y^{2}\right)^{4}-4 \lambda_{1}^{3} \lambda_{2}^{3}-\lambda_{1} \lambda_{2} \sigma\left(x-y^{2}\right)^{+}\left(x-y^{1}\right), \\
4 \tau\left(y^{1}-y^{2}\right)^{2} \tilde{f}_{13}^{-1}= & \lambda_{1}\left(\sigma-\lambda_{1}^{2}\right)\left(y^{1}-y^{2}\right)^{2}\left(x-y^{1}\right)^{+}-2 \lambda_{1}^{3} \lambda_{2}^{2}\left(y^{1}-y^{2}\right)^{+}, \\
4 \tau\left(y^{1}-y^{2}\right)^{2} \tilde{f}_{14}^{-1}= & -\lambda_{1} \lambda_{2}^{2}\left(y^{1}-y^{2}\right)^{2}\left(x-y^{1}\right)^{+}-2 \lambda_{1} \lambda_{2}^{2}\left(\tau-\lambda_{1}^{2}\right)\left(y^{1}-y^{2}\right)^{+}, \\
4 \tau \tilde{f}_{33}^{-1}= & 2 \tau\left(x-y^{1}\right)^{2}+\lambda_{1}^{2}\left(\tau-\lambda_{2}^{2}\right), \\
4 \tau \tilde{f}_{34}^{-1}= & \lambda_{1}^{2} \lambda_{2}^{2} .
\end{aligned}
$$

Matrix elements of $\tilde{b}^{+} \tilde{b}$ :

$$
\begin{aligned}
2 \tau\left(y^{1}-y^{2}\right)^{2} \tilde{b}^{+} \tilde{b}_{11} & =-\sigma^{2}+\lambda_{1}^{2}\left(3 \sigma-2 \lambda_{1}^{2}-2 \lambda_{2}^{2}\right), \\
2 \tau\left(y^{1}-y^{2}\right)^{2} \tilde{b}^{+} \tilde{b}_{12} & =\lambda_{1} \lambda_{2}\left(3 \sigma-2 \lambda_{1}^{2}-2 \lambda_{2}^{2}\right), \\
\tilde{b}^{+} \tilde{b}_{33} & =-1, \\
\tilde{b}^{+} \tilde{b}_{13}=\tilde{b}^{+} \tilde{b}_{14} & =\tilde{b}^{+} \tilde{b}_{34}=0 .
\end{aligned}
$$

\section{References}

[1] B. Berg and M. Lüscher, Nucl. Phys. B160 (1979) 281;

A.A. Belavin, V.A. Fateev, A.S. Schwarz and Yu.S. Tyupkin, Phys. Lett. 83B (1979) 317;

E. Corrigan, P. Goddard, H. Osborn and S. Templeton, Nucl. Phys. B159 (1979) 463;

H. Osborn, Nucl. Phys. B159 (1979) 497

[2] A. D'Adda and P. Di Vecchia, Phys. Lett. 73B (1978) 162

[3] B. Berg, unpublished 
[4] E. Corrigan, P. Goddard and S. Templeton, Nucl. Phys. B151 (1979) 93; V.G. Drinfeld and Yu.I. Manin, Yad. Fiz. 29 (1979) 1646

[5] I. Jack, Nucl. Phys. B174 (1980) 526

[6] V.A. Fateev, I.V. Frolov and A.S. Schwarz, Nucl. Phys. B154 (1979) 1;

B. Berg and M. Lüscher, Comm. Math. Phys. 69 (1979) 57

[7] G. 't Hooft, Phys. Rev. D14 (1976) 3432 (E: D18 (1978) 2199);

S. Chadha, A. D'Adda, P. Di Vecchia and F. Nicodemi, Phys. Lett. 67B (1977) 470; 72B (1977) 103;

F. Ore, Phys. Rev. D16 (1977) 2577

[8] L.S. Brown and D.B. Creamer, Phys. Rev. D18 (1978) 3695

[9] C. Berge, The theory of graphs and its applications (Methuen, London, 1966)

[10] N.H. Christ, E. Weinberg and N.K. Stanton, Phys. Rev. D18 (1978) 2013

[11] R. Jackiw, C. Nohl and C. Rebbi, Phys. Rev. D15 (1977) 1642 